\title{
The effect of asymmetric gas puffing on toroidal flow in the edge of tokamak plasma
}

\author{
Raghvendra Singh \\ Institute for Plasma Research, Bhat, Gandhinagar-3824 28, India \\ Andre Rogister \\ Institut für Plasmaphysik, Forschungszentrum Jülich GmbH, D-52425, Jülich, Germany \\ Predhiman Kaw \\ Institute for Plasma Research, Bhat, Gandhinagar-3824 28, India
}

(Received 1 August 2003; accepted 20 October 2003)

\begin{abstract}
The neoclassical theory of toroidal plasma rotation in the presence of an asymmetric neutral gas source in the edge of tokamak is examined. The poloidal dependence of momentum damping and ion energy loss due to charge-exchange processes are included. It is shown that the toroidal flow velocity increases significantly if the gas is fueled from the inboard side of the tokamak; the radial electric field and its shear are modified accordingly. It is also shown that for a fixed gas-fueling rate, the asymmetric fueling has a smaller role in TEXTOR [G. Mank et al., Phys. Rev. Lett. 85, 2312 (2000)] than in COMPASS-D [M. Valovi et al., Plasma Phys. Controlled Fusion 44, A175 (2002)]. The role of asymmetric gas injection is therefore effectively stronger in COMPASS-D. (C) 2004 American Institute of Physics. [DOI: 10.1063/1.1633558]
\end{abstract}

\section{INTRODUCTION}

It is now widely recognized that edge physics plays a key role in optimization of the global confinement performance of tokamak discharges. Several experiments have demonstrated that the transition from L-mode (low confinement) to H-mode (high confinement) can be affected by neutral atoms in the edge of tokamak plasma. ${ }^{1-6}$ The edge region always has a significant neutral density owing to recycling from the wall and from the limiter and/or the diverter plate region. In order to arrive at a detailed understanding of the role of neutral gas on the H-mode physics, the asymmetric gas puffing experiments have been performed in COMPASS-D (Refs. 4 and 5) and MAST. ${ }^{6}$ A lower H-mode power threshold is observed if the gas is fueled from the location of inboard mid-plane in comparison with the gas fueling from the outboard mid-plane. The object of this paper is to understand how a simple change in the location of the gas puff could reduce H-mode power threshold.

Gas puff with a strong poloidal asymmetry may influence the edge dynamics in two ways: (I) It could modify the local density and temperature profiles owing to the enhancement or reduction of atomic physics effects in edge instabilities, viz., the ion temperature gradient mode (ITG), ${ }^{7}$ high $m$-drift resistive ballooning mode (high- $m$ DRBM), ${ }^{8}$ etc. However the ionization and charge-exchange rates have very weak effects on the growth rates of edge instabilities because the ionization and charge-exchange rates $\left(\sim 10^{3}-10^{4} \mathrm{~s}^{-1}\right)$ are much smaller than the instability growth rates $\left(\sim 10^{5}-10^{6} \mathrm{~s}^{-1}\right)$. (II) It can also enhance the $\mathbf{E} \times \mathbf{B}$ shear rate which, it is well known, can suppress background turbulence ${ }^{9,10}$ if

$$
\gamma_{E}=\left|\frac{\Delta_{r} \partial \omega_{E}}{\partial r}\right| \geqslant \gamma_{T}
$$

here, $\omega_{E}=-k_{\theta} E_{r} / B$ is the Doppler shifted frequency associated with the $\mathbf{E} \times \mathbf{B}$ motion, $k_{\theta}$ is the poloidal wave number, $E_{r}$ is the radial electric field, $\Delta_{r}$ is the linear radial mode width of the background instability, $\gamma_{E}$ and $\gamma_{T}$ are the $\mathbf{E} \times \mathbf{B}$ shearing rate and the linear growth rate of the background instability, respectively. $E_{r}$ is given by the well known radial momentum balance equation in leading order:

$$
\begin{aligned}
E_{r} & =B_{\theta} U_{\varphi_{i}}-B_{\varphi} U_{\theta_{i}}+\frac{T_{i}}{e} \frac{\partial \ln P_{i}}{\partial r} \\
& \approx B_{\theta} U_{\varphi_{i}}+\left(-\kappa+1+\eta_{i}^{-1}\right) \frac{T_{i}}{e} \frac{\partial \ln T_{i}}{\partial r},
\end{aligned}
$$

where, according to standard neoclassical theory, the poloidal ion velocity $U_{\theta_{t}} \approx \kappa \partial_{r} T_{i} / e B_{\varphi}$, with $\kappa \approx 1.17$ in the low collision (banana) regime, $\kappa \approx-0.50$ in the intermediate (Plateau) collision regime and $\kappa \approx-1.83$ in the high collision (Pfirsch-Schlüter) regime; $U_{\varphi_{i}}$ is the ion toroidal flow velocity, $B_{\theta}$ and $B_{\varphi}$ are the poloidal and toroidal magnetic field components respectively; and $\partial_{r} P_{i}$ is the ion pressure gradient. Equation (2) suggests that the local $\mathbf{E} \times \mathbf{B}$ shear rate may be controlled either by the pressure gradient or by the toroidal rotation. Several authors ${ }^{11-15}$ have studied the generation of toroidal and poloidal flows in the framework of neoclassical theory without including the role of neutral atoms. Claassen et al. ${ }^{15}$ have derived the neoclassical toroidal and poloidal rotation velocities in high collisionality plasma by using Braginskii's ${ }^{18}$ two fluid equations including Mikhailovskii and Tsypin ${ }^{19}$ correction to ion stress tensor. The constant neutral charge exchange rate, which provides a drag force on ion flow, is also included in the analysis. It was 
shown that constant charge exchange-damping rate does not appreciably modify the toroidal flow ${ }^{15,16}$ and the transport coefficient. $^{20}$ Rogister et al. ${ }^{16}$ explained quite accurately the experimental results in edge localized mode (ELM)-free Ohmic H-mode discharge ${ }^{17}$ by using the neoclassical equations of toroidal flow, which were derived by Claassen et al. ${ }^{15}$ In leading order, the equation providing the PfirschSchlüter toroidal flow velocity is given by ${ }^{15,16}$

$$
\begin{aligned}
\partial_{r}\left(U_{\phi i}\right)_{\pi_{i}} & \approx 0.107 q^{2} \partial_{r} \ln T_{i}\left(B_{\phi} U_{\theta i} / B_{\theta}\right) \\
& \approx-0.2 q^{2}\left(T_{i} / e B_{\theta}\right)\left(\partial_{r} \ln T_{i}\right)^{2},
\end{aligned}
$$

where $q$ is the safety factor and $U_{\phi i}$ is the ion toroidal flow velocity. Catto et al. $^{21}$ have studied the importance of atomic physics processes on edge transport, within the framework of neoclassical theory. It is shown that neutral viscosity can significantly alter the electric field in the edge of tokamak if neutral viscosity dominates over standard ion viscosity, i.e., ${ }^{16}$ $\left(N_{n} / N_{i}\right)\left(c_{i} \lambda_{c x} / a_{i}^{2} \nu_{i}\right) \approx\left(N_{n} / N_{i}\right) \times 10^{3} \gg 1$. For $\left(N_{n} / N_{i}\right)$ $\times\left(c_{i} \lambda_{c x} / a_{i}^{2} \nu_{i}\right) \gg 1$ limit, Fülöp et al. ${ }^{22}$ have explored the effect of poloidal variation of neutral atoms on toroidal flow velocity through neutral viscosity. With small inverse aspect ratio and circular cross section, they derived the neoclassical equation of toroidal flow given as

$$
\begin{aligned}
\left(U_{\phi i}\right)_{\pi_{n}} & =\left(2 \epsilon R_{0} \partial_{\psi} T_{i} / e\right)\left[k \cos \theta+(1-k) \cos \theta_{*}\right] \\
& \approx\left(2 \epsilon T_{i} / e B_{\theta}\right)\left(\partial_{r} \ln T_{i}\right)
\end{aligned}
$$

where $\epsilon=r / R$ is the inverse aspect ratio. Note that the neoclassical toroidal flow Eq. (3) is derived from flux average ion viscous tensor while toroidal flow Eq. (4) results from flux average neutral viscous tensor. For $\partial_{r} \ln T_{i} \sim \partial_{r} \ln U_{\phi i}$, we can compare the toroidal flow velocities resulting from ion viscous tensor ${ }^{15,16}$ and from neutral viscous tensor, ${ }^{22}$ respectively. The comparison shows that $\left(U_{\phi i}\right)_{\pi_{i}} /\left(U_{\phi i}\right)_{\pi_{n}}$ $\approx q^{2} / 20 \epsilon \geqslant 1$. From this comparison we conclude that even when conditions are such that the neutral viscous tensor related terms are dominant i.e., $\left(N_{n} / N_{i}\right) \times 10^{3} \geqslant 1$, the contribution to generation of flow coming from the ion viscous tensor may not be ignorable. It thus becomes important that a calculation retaining the contributions from both stress tensors on an equal footing should be attempted.

In this paper we have attempted such a calculation. Unlike Catto et al. ${ }^{21}$ and Fülöp et al. ${ }^{22}$ we do not start our analysis with the equation of motion obtained by summing the equations of neutral and ion fluids. Instead, following Rogister ${ }^{11}$ we write an equation of motion for the ion fluid only and take account of effects due to the neutral stress tensor by retaining the effects of charge exchange terms on momentum and energy loss of ions. For simplicity, the neutral density profile in the presence of a poloidally asymmetric gas puff is assumed to be of the form $N_{n}=N_{0 n}$ (1 $\left.+\Delta_{1} \cos \chi\right) \equiv N_{0 n}[1+g(\chi)]$, where $N_{0 n}$ is the averaged neutral density, $\Delta_{1}$ is the degree of asymmetry due to gas refueling ( $\Delta_{1}=\Delta_{1}$ at $\chi=0$ corresponds to the out board side, $\Delta_{1}$ $=-\Delta_{1}$ at $\chi=\pi$ corresponds to the in board side), and $g(\chi)=\Delta_{1} \cos \chi$. Toroidal symmetry is assumed to be preserved.
The basic physics of the generation of neoclassical toroidal and poloidal rotation velocities in high collisionality plasmas goes as follows. In a tokamak, the magnetic field lines spiral around the minor axis and the Pfirsch-Schlüter current along field lines is unable to neutralize the space charge produced along a given surface by magnetic curvature and grad $\mathbf{B}$ drifts, because of finite resistivity effects. This creates poloidally $(\chi)$ asymmetric plasma density, temperature, and parallel ion flow. Under these conditions, the flux surface averaged toroidal component of $\boldsymbol{\nabla} \cdot \stackrel{\pi}{i}_{i}$ results in a large toroidal rotation. In the present problem, the poloidal asymmetry in the plasma is introduced additionally by asymmetric neutral gas fueling and this also contributes to flux surface averaged $\boldsymbol{\nabla} \cdot \overleftrightarrow{\pi}_{i}$ in a manner similar to PfirschSchlüter problem thereby contributing to additional toroidal flows in the plasma.

The remainder of the paper is organized as follows. The basic equations and coordinates system are given in Sec. II. In Sec. III, the general formulation of neoclassical toroidal flow and ordering of various terms are given. In Sec. IV, we derive the toroidal flow equations in large aspect ratio limit. After briefly summarizing our results, we discuss the applications of our theory in Sec. V.

\section{BASIC EQUATION}

We use Braginskii's two fluid equations ${ }^{18}$ including the Mikhailovskii and Tsypin corrections of the stress tensor. ${ }^{19}$ The ion continuity, momentum, and energy equations are, respectively,

$$
\begin{aligned}
& \frac{\partial N_{i}}{\partial t}+\boldsymbol{\nabla} \cdot N_{i} \mathbf{U}_{i}=0, \\
& m_{i} N_{i}\left(\frac{\partial}{\partial t}+\mathbf{U}_{i} \cdot \boldsymbol{\nabla}\right) \mathbf{U}_{i}=-\boldsymbol{\nabla} P_{i}-\boldsymbol{\nabla} \cdot \boldsymbol{\pi}_{i}+e N_{i}\left(\mathbf{E}+\mathbf{U}_{i} \times \mathbf{B}\right) \\
& -M_{c x}(\chi) \\
& \frac{3}{2} N_{i}\left(\frac{\partial}{\partial t}+\mathbf{U}_{i} \cdot \boldsymbol{\nabla}\right) T_{i}+P_{i} \boldsymbol{\nabla} \cdot \mathbf{U}_{i}=-\boldsymbol{\nabla} \cdot \mathbf{q}_{i}-E_{i}(\chi),
\end{aligned}
$$

where $\boldsymbol{\pi}_{i}$ is the ion stress tensor given by Braginskii ${ }^{18}$ and Mikhailovskii and Tsypin ${ }^{19}$ (see Appendix A), $M_{c x}(\chi)$ and $E_{i}(\chi)$ describe the effect associated with atomic processes, viz., ion momentum loss to the wall via charge exchange with neutrals and likewise energy loss via charge exchange processes, respectively, $\quad \mathbf{q}_{i}=-\kappa_{\| i} \nabla_{\|} T_{i}-\kappa_{\perp i} \nabla_{\perp} T_{i}+\kappa_{x i} \hat{n}$ $\times \nabla T_{i}$ is the diffusive thermal flux, $\kappa_{\| i}=3.9\left(N_{i} T_{i} / m_{i} \nu_{i}\right)$ and $\kappa_{\perp i}=2 N_{i} T_{i} / m_{i} \Omega_{i}^{2}$ are the parallel and perpendicular diffusion coefficient, respectively and $\kappa_{x i}=5 N_{i} T_{i} / 2 m_{i} \Omega_{i}$; other notations are standard. For simplicity, here we have neglected the effects of poloidally dependence of ionization processes on the generation of angular momentum density (discussion will be included in a forthcoming paper). Experimental observations by Valoviè et al. ${ }^{4}$ indeed suggest that for both the inboard and the outboard gas puff, ionization occurs in the scrape off layer since the density profile within the experimental resolution remain the same in both cases.

The coordinate system $(\hat{p}, \hat{b}, \hat{n})$ is tied to the magnetic field, $\hat{n}=\mathbf{B} / B$ is the unit vector along the magnetic field 
lines, $\hat{p}$ is the orthogonal to the magnetic surface, and $\hat{b}$ $=\hat{n} \times \hat{p}$. The unit vectors $\hat{p}, \hat{b}, \hat{n}$ are related to the flux coordinates $\hat{e}_{\psi}, \hat{e}_{\chi}, \hat{e}_{\phi}$ (where $\psi$ is the poloidal magnetic flux, $\chi$ the generalized poloidal angle and $\phi$ is the toroidal angle) by

$$
\begin{aligned}
& \hat{p}=\hat{e}_{\psi}, \quad \hat{b}=\left(\frac{B_{\phi}}{B}\right) \hat{e}_{\chi}-\left(\frac{B_{\chi}}{B}\right) \hat{e}_{\phi}, \\
& \hat{n}=\left(\frac{B_{\chi}}{B}\right) \hat{e}_{\chi}+\left(\frac{B_{\phi}}{B}\right) \hat{e}_{\phi} .
\end{aligned}
$$

We note that these notations can easily be identified with the standard tokamak coordinates system ( $r$, radial; $\theta$, poloidal; $\phi$, toroidal) in the limit of a large aspect ratio tokamak with circular cross section and toroidal symmetry $(\partial / \partial \phi \equiv 0)$. The differential operators can be written as

$$
\begin{aligned}
& \hat{p} \cdot \boldsymbol{\nabla} \equiv h_{\psi}^{-1} \frac{\partial}{\partial \psi} \sim \frac{\partial}{\partial r} \sim L_{\psi}^{-1}, \\
& \hat{b} \cdot \boldsymbol{\nabla} \equiv\left(\frac{B_{\phi}}{B}\right) h_{\chi}^{-1} \frac{\partial}{\partial \chi} \sim \frac{1}{r} \frac{\partial}{\partial \theta} \sim r^{-1}, \\
& \hat{n} \cdot \boldsymbol{\nabla} \equiv\left(\frac{B_{\chi}}{B}\right) h_{\chi}^{-1} \frac{\partial}{\partial \chi} \sim \frac{1}{q R} \frac{\partial}{\partial \theta} \sim(q R)^{-1},
\end{aligned}
$$

where $h_{\psi}=1 / h_{\phi} B_{\chi}, h_{\chi}=J B_{\chi}, J=h_{\psi} h_{\chi} h_{\phi}$ is the Jacobian of the transformation $(\mathbf{r} \rightarrow \psi, \chi, \phi), \nu=h_{\chi} B_{\phi} / h_{\phi} B_{\chi} \sim r B_{\phi} / R B_{\chi}$ is the pitch of the field lines, $q=\oint \nu d \chi$ is the safety factor. We note that $h_{\phi}=R_{0}(1+\epsilon \cos \chi)$ and $B_{\phi}=B_{\phi 0}(1-\epsilon \cos \chi)$ in the case of the large aspect ratio tokamak with circular cross section, where $\epsilon=r / R_{0}$ is the inverse aspect ratio, $r$, and respectively, $R_{0}$ the minor and major radii. In this manuscript we use the notations and ordering as given in the paper by Rogister ${ }^{11}$ unless otherwise mentioned.

\section{GENERAL FORMULATION OF NEOCLASSICAL TOROIDAL FLOW}

Summing up the toroidal component of the ion and electron momentum equations yields the radial current density

$$
J_{\psi}=\frac{1}{B_{\chi}} \hat{e}_{\phi} \cdot\left[\boldsymbol{\nabla} \cdot \pi_{i}+m_{i} N_{i}\left(\frac{\partial}{\partial t}+\mathbf{U}_{i} \cdot \boldsymbol{\nabla}\right) \mathbf{U}_{i}+M_{c x}(\psi, \chi) .\right.
$$

The continuity Eq. (5) in a general coordinate system is given as

$$
\frac{\partial N_{i}}{\partial t}+J^{-1}\left[\frac{\partial}{\partial \psi}\left(h_{\chi} h_{\phi} N_{i} U_{\psi}\right)+\frac{\partial}{\partial \chi}\left(h_{\psi} h_{\phi} N_{i} U_{i \chi}\right)\right]=0 .
$$

Similarly the convective term in the momentum Eq. (10) can be expressed as

$$
\begin{aligned}
N_{i} \hat{e}_{\phi} \cdot\left[\left(\mathbf{U}_{i} \cdot \boldsymbol{\nabla}\right) \mathbf{U}_{i}\right]= & -J^{-1} U_{\phi i}\left[\frac{\partial}{\partial \psi}\left(h_{\chi} h_{\phi} N_{i} U_{\psi i}\right)\right. \\
& \left.+\frac{\partial}{\partial \chi}\left(h_{\psi} h_{\phi} N_{i} U_{\chi i}\right)\right] \\
& +\left(J h_{\phi}\right)^{-1}\left[\frac{\partial}{\partial \psi}\left(h_{\chi} h_{\phi}^{2} U_{\psi i} U_{\phi i}\right)\right. \\
& \left.+\frac{\partial}{\partial \chi}\left(h_{\psi} h_{\phi}^{2} U_{\chi i} U_{\phi i}\right)\right] .
\end{aligned}
$$

Here we have made use of a tensorial relation $\boldsymbol{\nabla} \hat{e}_{\phi}$ $=-\hat{e}_{\phi} \boldsymbol{\nabla} \ln h_{\phi}$. Inserting Eq. (11) and Eq. (12) into Eq. (10), and integrating the resulting expression over magnetic flux surfaces leads to the relation

$$
\begin{aligned}
\oint J_{\psi} h_{\chi} h_{\phi} \frac{d \chi}{2 \pi}= & \oint J h_{\phi} \frac{d \chi}{2 \pi}\left[\frac{\partial\left(m_{i} N_{i} U_{\phi_{i}}\right)}{\partial t}+M_{c x}(\psi, \chi)\right. \\
& +\oint \hat{e}_{\phi} \cdot\left(\nabla \cdot \pi_{i}\right) J h_{\phi} \frac{d \chi}{2 \pi} \\
& +\frac{\partial}{\partial \psi} \oint J h_{\phi} h_{\psi}^{-1} m_{i} N_{i} U_{\psi i} U_{\phi i} \frac{d \chi}{2 \pi}
\end{aligned}
$$

where the last term corresponds to the radial angular momentum flux. The latter includes not only a neoclassical contribution, which is generally negligible, but also an anomalous one:

$$
U_{\psi i}\left(U_{\phi i}\right)=\left(U_{\psi i}\right)_{\text {neo }} U_{\phi i}+\left\langle u_{\psi i} u_{\phi i}\right\rangle,
$$

where the angular brackets \langle\rangle represents an average over the small time and space scales of the turbulence; and $u_{\psi i}$, respectively $u_{\phi i}$ are the radial and toroidal components of the perturbed flow velocity associated with background turbulence; at the plasma edge, the latter may be driven, e.g., by the ion temperature gradient (ITG) or the high- $m$ drift resistive ballooning (DRB) instability. In deriving Eq. (13), we have made use of the ion continuity equation where the momentum convection term, i.e., Eq. (12) can be expressed as

$$
\begin{aligned}
\oint \frac{h_{\chi} h_{\phi}}{B_{\chi}} N \hat{e}_{\phi} \cdot(\mathbf{U} \cdot \nabla \mathbf{U}) d \chi= & -\oint J h_{\phi} U_{\phi} \boldsymbol{\nabla} \cdot(N \mathbf{U}) d \chi \\
& +\frac{\partial}{\partial \psi} \oint J h_{\phi} h_{\psi}^{-1} N_{i} U_{\psi i} U_{\varphi i} d \chi .
\end{aligned}
$$

To compare the magnitude of the various terms, we consider the scaling relevant to the edge of the tokamak's edge ${ }^{11}$

$$
\frac{r}{q R} \sim \frac{c_{i}}{q R \nu_{i}} \sim \frac{L_{\psi}}{r} \sim\left(\frac{m_{e}}{m_{i}}\right)^{1 / 4} \sim\left(\frac{a_{i}}{L_{\psi}}\right)^{1 / 2} \sim \mu \ll 1,
$$

and

$$
\nu_{c x} \sim \nu_{\text {ion }} \leqslant \nu_{i} ; \quad L_{\psi} \sim L_{N, T},
$$

where $\nu_{i}$ is the ion collision frequency, $L_{\psi} \sim L_{N, T}$ the plasma density or temperature gradient scale, $m_{i}\left(m_{e}\right)$ the ion (electron) mass, $c_{i}=\sqrt{T_{i} / m_{i}}$ the ion thermal velocity, $a_{i}=c_{i} / \Omega_{i}$ 
the ion Larmor radius, $\Omega_{i}=e B / m_{i}$ the ion cyclotron frequency, and $\mu$ the small expansion parameter.

We first evaluate the stress tensor term in Eq. (13); the $\stackrel{\pi}{\pi}_{i}$ can be split into a diagonal $\left(\stackrel{\leftrightarrow}{\pi}_{0, i}\right)$ and an off-diagonal $\left(\stackrel{\pi}{\pi}_{3-4, i}, \overleftrightarrow{\pi}_{1-2, i}\right)$ part, i.e.,

$$
\overleftrightarrow{\pi}_{i}=\stackrel{\pi}{\pi i}_{0 i}+\overleftrightarrow{\pi}_{3-4, i}+\stackrel{\leftrightarrow}{\pi}_{1-2, i}
$$

We note that all these tensors are of the form

$$
\stackrel{\leftrightarrow}{\pi}=\boldsymbol{\alpha} \boldsymbol{\beta}+\boldsymbol{\beta} \boldsymbol{\alpha} .
$$

It can be shown that

$$
\oint J h_{\phi} \hat{e}_{\phi} \cdot(\boldsymbol{\nabla} \cdot \stackrel{\leftrightarrow}{\pi}) d \chi=\frac{\partial}{\partial \psi} \oint J h_{\phi} h_{\chi}^{-1} \pi_{\psi \phi} d \chi
$$

The contribution to Eq. (19) from the tensor $\left(\stackrel{\pi}{\pi}_{0 i}\right)$ vanishes (the latter is diagonal), and the contribution from the off diagonal tensor can be written in leading order as

$$
\begin{aligned}
\oint \hat{e}_{\phi} \cdot\left(\nabla \cdot \pi_{i}\right) J h_{\phi} d \chi= & \frac{\partial}{\partial \psi} \oint h_{\psi}^{-1} J h_{\phi} d \chi\left(\pi_{3-4, i}\right)_{\psi \phi}+\frac{\partial}{\partial \psi} \oint h_{\psi}^{-1} J h_{\phi} d \chi\left(\pi_{1-2, i}\right)_{\psi \phi} \\
= & -\frac{m_{i}}{e} \frac{\partial}{\partial \psi}\left\{h_{\phi}^{2} B_{\phi}^{2} \oint d \chi\left[\frac{P_{i}}{B^{4}} \frac{\partial}{\partial \chi}\left(U_{\| i} B\right)+\frac{8}{5} \frac{q_{\| i}}{B^{4}} \frac{\partial B}{\partial \chi}\right]\right\} \\
& -\frac{12}{10} \frac{m_{i}}{e} \frac{\partial}{\partial \psi}\left\{h_{\phi}^{2} B_{\phi}^{2} \oint d \chi\left[\frac{\nu_{i}}{\Omega_{i}} \frac{B_{\chi} J}{B_{\phi}} \frac{P_{i}}{B^{2}} h_{\psi}^{-1} \frac{\partial U_{\| i}}{\partial \psi}\right]\right\} .
\end{aligned}
$$

Here, the contribution proportional to $q_{\| i}=\kappa_{\| i} \hat{n} \cdot \nabla T_{i}$ arises from the thermal stresses (see Mikhailovskii and Tsypin ${ }^{19}$ ).

We expand the equilibrium quantities in powers of the inverse aspect ratio and seek solutions of the form

$$
\begin{aligned}
& F(\psi, \chi)=F^{(0)}(\psi)\left[1+\mu f^{(1)}(\psi, \chi)+O\left(\mu^{2}\right)\right], \\
& B / B_{0}=(1-\epsilon \cos \chi) ; \quad b^{(1)}(\psi, \chi) \equiv-\epsilon \cos \chi,
\end{aligned}
$$

where $F(\psi)=N_{i}, T_{i}^{(0)}, \Phi$ or $B$ and $f(\psi, \chi)=n_{i}, t_{i}, \phi$ or $b$. We note that

$$
\begin{aligned}
& \mathbf{M}_{c x}^{(0)}(\psi, \chi)=\mathbf{M}_{c x}^{(0)}(\psi)[1+g(\chi)], \\
& E_{i}(\psi, \chi)=E_{i}^{(0)}(\psi)[1+g(\chi)],
\end{aligned}
$$

where $\mathbf{M}_{c x}^{(0)}(\psi)=m_{i} N_{i}^{(0)} \nu_{c x}^{(0)} \mathbf{U}_{i}^{(0)}, \quad E_{i}(\psi)=(3 / 2) N_{i} T_{i} \nu_{c x}^{(0)}$, $\nu_{c x}^{(0)}$ is the charge exchange rate coefficient, and $\oint g(\chi) d \chi$ $=0$.

We order the ion flow velocities with respect to the ion thermal velocity as follows: ${ }^{11}$

$$
U_{\phi i} \sim \mu c_{i}, \quad U_{\chi i} \sim \mu^{2} c_{i}, \quad U_{\psi i} \approx 0 .
$$

Substituting Eqs. (20)-(21) into Eq. (13), we get

$$
\begin{aligned}
\oint J h_{\phi} \frac{d \chi}{2 \pi}\left[\frac{\partial\left(m_{i} N_{i}^{(0)} U_{\phi_{i}}^{(0)}\right)}{\partial t}+m_{i} N_{i}^{(0)} \nu_{c x}^{(0)} U_{\phi_{i}}^{(0)}\right. \\
\left.+\frac{\partial}{\partial \psi} \oint J h_{\phi} h_{\psi}^{-1} m_{i} N_{i}\left\langle\tilde{u}_{E, r} \tilde{u}_{\phi i}\right\rangle \frac{d \chi}{2 \pi}\right] \\
=\frac{m_{i}}{e} h_{\phi}^{2} B_{\phi}^{2} \frac{\partial}{\partial \psi}\left\{\frac { P _ { i } ^ { ( 0 ) } } { B _ { 0 } ^ { 3 } } \oint \left[\left(p_{i}^{(1)}-4 b^{(1)}\right)\right.\right. \\
\times\left(\frac{\partial u_{\| i}^{(1)}}{\partial \chi}+U_{\| i}^{(0)} \frac{\partial b^{(1)}}{\partial \chi}\right)
\end{aligned}
$$

$$
\begin{aligned}
& \left.-1.6 \chi_{\| i}^{(0)} \frac{B_{\chi}^{(0)}}{B_{0}} \frac{1}{h_{\chi}^{(0)}}\left(\frac{\partial t_{i}^{(1)}}{\partial \chi} \frac{\partial b^{(1)}}{\partial \chi}\right)\right] \frac{d \chi}{2 \pi} \\
& \left.+\frac{12}{10} \frac{P_{i}^{(0)}}{B_{0}^{2}} \frac{\nu_{i}^{(0)}}{\Omega_{i}^{(0)}} \frac{h_{\chi}^{(0)}}{B_{\phi 0}} \frac{1}{h_{\psi}^{(0)}} \frac{\partial U_{\| i}^{(0)}}{\partial \psi}\right\},
\end{aligned}
$$

where $p_{i}^{(1)}=p_{j} / P_{j}^{(0)}, \quad n_{j}^{(1)}=n_{j} / N_{j}^{(0)}, \quad t_{j}^{(1)}=t_{j} / T_{j}^{(0)}, \quad$ and $\phi^{(1)}=e \phi / T_{e}^{(0)}$ are the normalized perturbed variables, $\chi_{\| i}$ $=3.9 T_{i}^{(0)} / m_{i} \nu_{i}^{(0)}$ and $\nu_{i}^{(0)}$ is the ion collision frequency. Here the torque due to external radial current is neglected assuming no current is drawn by externally polarizing the plasma. In view of large electron mobility along the magnetic field lines $\left(m_{e} / m_{e} \rightarrow 0\right)$ the electron parallel momentum equation and the energy equation yield the electron adiabatic relations

$$
n_{e}^{(1)}=\phi^{(1)}, \quad t_{e}^{(1)}=0 .
$$

The parallel component of the ion momentum equation (6) reads in leading order

$$
\hat{n} \cdot\left[\nabla P_{i}+e N_{i} \nabla \phi+M_{c x}(\psi, \chi)\right]=0 .
$$

From Eqs. (18) and (25), we get

$$
\begin{aligned}
\frac{\partial}{\partial \chi}[ & \left.P_{i}^{(0)}\left(n^{(1)}+t_{i}^{(1)}\right)+P_{i}^{(0)} \phi^{(1)}\right] \\
& +m_{i} N_{i}^{(0)}\left(\frac{h_{\chi} B}{B_{\chi}}\right) \nu_{c x}^{(0)} g(\chi) U_{\|}^{(0)}=0
\end{aligned}
$$

or, in view of the adiabatic relation (24):

$$
\frac{\partial t_{i}^{(1)}}{\partial \chi}=-2 \frac{\partial n^{(1)}}{\partial \chi}-\frac{m_{i} N_{i}^{(0)}}{P_{i}^{(0)}}\left(\frac{h_{\chi} B}{B_{\chi}}\right) \nu_{c x}^{(0)} g(\chi) U_{\|}^{(0)},
$$

where $n_{i}^{(1)}=n_{e}^{(1)}=n^{(1)}$. Equation (26) shows that the total electron and ion pressure along the field lines is not constant in the presence of an asymmetric neutral source. 
The perpendicular components of the ion momentum equation (6) and the continuity equation (5) read, in leading order:

$$
\begin{aligned}
\hat{b} \cdot \mathbf{U}_{i} & =U_{\beta i}=\frac{1}{e B N_{i}} h_{\psi}^{-1}\left(\frac{\partial}{\partial \psi} P_{i}+e N_{i} \frac{\partial \phi}{\partial \psi}\right), \\
\hat{p} \cdot \mathbf{U}_{i} & =U_{\psi i}=-\frac{1}{e B N_{i}}\left(\frac{B \phi}{B}\right) h_{\chi}^{-1}\left(\frac{\partial}{\partial \chi} P_{i}+e N_{i} \frac{\partial \phi}{\partial \chi}\right),
\end{aligned}
$$

and

$$
\frac{1}{J} \frac{\partial}{\partial \chi}\left(h_{\psi} h_{\phi} N_{i} U_{\chi i}\right)=0,
$$

where the poloidal flow velocity $U_{\chi i}=\left(B_{\phi} / B\right) U_{\beta_{i}}$ $+\left(B_{\chi} / B\right) U_{\| i}$, and $U_{\|}=\hat{n} \cdot \mathbf{U}_{i}$. Combining Eqs. (27) and (28), we obtain

$$
\frac{\partial}{\partial \chi}\left(\frac{N_{i} U_{\| i}}{B}\right)=-\left(\frac{h_{\phi} B_{\phi}}{e}\right) \frac{\partial}{\partial \chi}\left[\frac{1}{B^{2}}\left(\frac{\partial P_{i}}{\partial \psi}+e N_{i} \frac{\partial \phi}{\partial \psi}\right)\right] .
$$

Equation (29) provides the relation between $\partial u_{\| i}^{(1)} / \partial \chi, n^{(1)}$ and $b^{(1)}$. It follows by combining Eqs. (21), (26), and (29) that

$$
\begin{aligned}
\frac{\partial u_{\| i}^{(1)}}{\partial \chi}= & -\frac{B_{0}}{e N^{(0)}} h_{\phi} B_{\phi} \frac{\partial F^{(1)}}{\partial \chi}-U_{\| i}^{(0)} \frac{\partial}{\partial \chi}\left(n^{(1)}-b^{(1)}\right) \\
& +\left\{( \frac { \nu _ { c x } ^ { ( 0 ) } } { \Omega _ { i } } ) ( \frac { B _ { \phi } } { B _ { \chi } } ) ( \frac { h _ { \chi } B } { B _ { \chi } } ) h _ { \psi } ^ { - 1 } \left[U_{\| i}^{(0)} \frac{\partial \ln N^{(0)}}{\partial \psi}\right.\right. \\
& \left.\left.+\frac{\partial U_{\| i}^{(0)}}{\partial \psi}\right]\right\} g(\chi),
\end{aligned}
$$

where $\partial F^{(1)} / \partial \chi$ can be written as

$$
\begin{aligned}
\frac{\partial F^{(1)}}{\partial \chi}= & \frac{P_{i}^{(0)}}{B_{0}^{2}}\left[\left(\frac{e}{T_{i}} \frac{\partial \phi^{(0)}}{\partial \psi}-\frac{\partial \ln N^{(0)}}{\partial \psi}\right) \frac{\partial n^{(1)}}{\partial \chi}\right. \\
& \left.-2\left\{\frac{e}{T_{i}} \frac{\partial \phi^{(0)}}{\partial \psi}+\left(1+\eta_{i}\right) \frac{\partial \ln N^{(0)}}{\partial \psi}\right\} \frac{\partial b^{(1)}}{\partial \chi}\right] .
\end{aligned}
$$

We now derive the relation between the density $n^{(1)}$, the temperature $t_{i}^{(1)}$, and magnetic perturbations $b^{(1)}$ from the ion energy equation (7). We obtain

$$
\begin{aligned}
& -\left[\frac{1}{B_{0}} h_{\psi}^{-1} \frac{\partial \phi^{(0)}}{\partial \psi}+\left(\frac{3}{2} \eta_{i}-1\right)\left(\frac{T_{i}^{(0)}}{e B_{0}}\right) h_{\psi}^{-1} \frac{\partial \ln N^{(0)}}{\partial \psi}\right. \\
& \left.+\left(\frac{B_{\chi}^{(0)}}{B_{\phi}^{(0)}}\right) U_{\| i}^{(0)}\right] \frac{B_{\phi}}{B_{0}} h_{\chi}^{-1} \frac{\partial n^{(1)}}{\partial \chi} \\
& +\frac{3}{2}\left[\frac{1}{B_{0}} h_{\psi}^{-1} \frac{\partial \phi^{(0)}}{\partial \psi}+\left(\frac{B_{\chi}^{(0)}}{B_{\phi}^{(0)}}\right) U_{\| i}^{(0)}\right]\left(\frac{B_{\phi}^{(0)}}{B_{0}}\right) h_{\chi}^{-1} \frac{\partial t_{i}^{(1)}}{\partial \chi} \\
& -5\left(\frac{T_{i}^{(0)}}{e B_{0}}\right)\left(h_{\psi}^{-1} \frac{\partial \ln T_{i}^{(0)}}{\partial \psi}\right) \frac{B_{\phi}}{B_{0}} h_{\chi}^{-1} \frac{\partial b^{(1)}}{\partial \chi} \\
& =\chi_{\| i}^{(0)}\left(\frac{B_{\chi}^{2}}{B_{\phi}^{2}}\right) h_{\chi}^{-2} \frac{\partial^{2} t_{i}^{(1)}}{\partial \chi^{2}}-\frac{3}{2} \nu_{c x}^{(0)} g(\chi) .
\end{aligned}
$$

Inserting the expression for $\partial t_{i}^{(1)} / \partial \chi$ obtained in (26) into Eq. (32), we obtain the following relation between $n^{(1)}$ and $b^{(1)}$

$$
\begin{aligned}
{\left[\frac{4}{B_{0}} h_{\psi}^{-1}\right.} & \frac{\partial \phi^{(0)}}{\partial \psi}+\left(\frac{3}{2} \eta_{i}-1\right)\left(\frac{T_{i}^{(0)}}{e B_{0}}\right) h_{\psi}^{-1} \frac{\partial \ln N^{(0)}}{\partial \psi} \\
& \left.+4\left(\frac{B_{\chi}}{B_{\phi}^{(0)}}\right) U_{\| i}^{(0)}\right] \frac{B_{\phi}^{(0)}}{B_{0}} h_{\chi}^{-1} \frac{\partial n^{(1)}}{\partial \chi}+\frac{3}{2}\left[\frac{1}{B_{0}} h_{\psi}^{-1} \frac{\partial \phi^{(0)}}{\partial \psi}\right. \\
& \left.+\left(\frac{B_{\chi}^{(0)}}{B_{(0)}}\right) U_{\| i}^{(0)}\right] \frac{m_{i} N^{(0)}}{P_{i}^{(0)}}\left(\frac{B_{\phi}^{(0)}}{B_{\chi}^{(0)}}\right) \nu_{c x}^{(0)} U_{\| i}^{(0)} g(\chi) \\
& +5\left(\frac{T_{i}^{(0)}}{e B_{0}}\right) h_{\psi}^{-1} \frac{\partial \ln T_{i}^{(0)}}{\partial \psi}\left(\frac{B_{\phi}^{(0)}}{B_{0}}\right) h_{\chi}^{-1} \frac{\partial b^{(1)}}{\partial \chi} \\
= & 2 \chi_{\| i}^{(0)}\left(\frac{B_{\chi}^{2}}{B_{0}^{2}}\right) h_{\chi}^{-2} \frac{\partial^{2} n^{(1)}}{\partial \chi^{2}} \\
& +\chi_{\| i}^{(0)}\left(\frac{B_{\chi}^{2}}{B_{0}^{2}}\right) \frac{m_{i} N^{(0)}}{P_{i}^{(0)}}\left(\frac{h_{\chi} B_{0}}{B_{\chi}}\right) \nu_{c x}^{(0)} U_{\| i}^{(0)} h_{\chi}^{-2} \frac{\partial g(\chi)}{\partial \chi} \\
& +\frac{3}{2} \nu_{c x}^{(0)} g(\chi)
\end{aligned}
$$

We have thus obtained four linear equations (23), (26), (30), (33) for four quantities $u_{\| i}^{(1)}(\chi), n^{(1)}(\chi), t_{i}^{(1)}(\chi)$, and $b^{(1)}$ $\times(\chi)$. Those are solved below under the assumptions of large aspect ratio with circular cross section geometry.

\section{TOROIDAL FLOW IN A LARGE ASPECT RATIO LIMIT}

We first write Eqs. (23), (26), (30), and (33) for large aspect ratio tokamaks with circular cross sections, in which case $\chi=\theta$ and $b^{(1)}=-\epsilon \cos \theta$.

Equations (23), (26), and (30) become, respectively,

$$
\begin{gathered}
\frac{\partial\left(m_{i} N_{i}^{(0)} U_{\phi_{i}}^{(0)}\right)}{\partial t}+m_{i} N_{i}^{(0)} \nu_{c x}^{(0)} U_{\phi_{i}}^{(0)}+\frac{\partial}{\partial r}\left(m_{i} N_{i}^{(0)}\left\langle\tilde{u}_{E r} \tilde{u}_{\phi i}\right\rangle\right) \\
=\left(\frac{m_{i} B_{\phi}^{2}}{e r}\right) \frac{\partial}{\partial r}\left\{\frac { P _ { i } ^ { ( 0 ) } } { B _ { 0 } ^ { 3 } } \oint \left[( n ^ { ( 1 ) } + t _ { i } ^ { ( 1 ) } - 4 b ^ { ( 1 ) } ) \left(\frac{\partial u_{\| i}^{(1)}}{\partial \theta}\right.\right.\right. \\
\left.\left.+U_{\| i}^{(0)} \frac{\partial b^{(1)}}{\partial \theta}\right)-1.6 \frac{\chi_{\| i}^{(0)}}{q R}\left(\frac{\partial b^{(1)}}{\partial \theta} \frac{\partial t_{i}^{(1)}}{\partial \theta}\right)\right] \frac{d \theta}{2 \pi} \\
\left.\quad+\frac{12}{10} \frac{P_{i}^{(0)}}{B_{0}^{2}} \frac{\nu_{i}^{(0)}}{\Omega_{i}^{(0)}} \frac{r}{B_{\phi 0}} \frac{\partial U_{\| i}^{(0)}}{\partial r}\right\}, \\
\frac{\partial t_{i}^{(1)}}{\partial \theta}=-2 \frac{\partial n^{(1)}}{\partial \chi}-\frac{m_{i} \nu_{c x}^{(0)}}{T_{i}^{(0)}} U_{\|}^{(0)} \Delta_{1} q R \cos \theta
\end{gathered}
$$

or

$t_{i}^{(1)}=-2 n^{(1)}-\frac{m_{i} \nu_{c x}^{(0)}}{T_{i}^{(0)}} U_{\|}^{(0)} \Delta_{1} q R \sin \theta$, 
and

$$
\begin{aligned}
\frac{\partial u_{\| i}^{(1)}}{\partial \theta} \approx \frac{\partial u_{\phi i}^{(1)}}{\partial \theta}= & -\left(\frac{B_{\phi}}{B_{\theta}}\right)\left[U_{E}^{(0)}-U_{* i}^{(0)}+\left(\frac{B_{\theta}}{B_{\phi}}\right) U_{\| i}^{(0)}\right] \frac{\partial n^{(1)}}{\partial \theta} \\
& +\left(\frac{B_{\phi}}{B_{\theta}}\right)\left[2 U_{E}^{(0)}+2\left(1+\eta_{i}\right) U_{* i}^{(0)}\right. \\
& \left.+\left(\frac{B_{\theta}}{B_{\phi}}\right) U_{\| i}^{(0)}\right] \frac{\partial b^{(1)}}{\partial \theta}+\frac{\nu_{c x}^{(0)}}{\Omega_{i}}\left(\Delta_{1} q R \frac{\partial \ln N^{(0)}}{\partial r}\right) \\
& \times\left(\frac{B_{\phi}}{B_{\theta}} U_{\| i}^{(0)}\right)\left(1+\frac{\partial \ln U_{\| i}^{(0)}}{\partial \ln N^{(0)}}\right) \cos \theta \\
& +\Delta_{1} q R \nu_{\text {ion }}^{(0)} \cos \theta
\end{aligned}
$$

where $u_{\| i}^{(1)} \approx u_{\phi i}^{(1)}, U_{E}^{(0)}=-E_{r} / B_{0}$ is the $\mathbf{E} \times \mathbf{B}$ drift velocity and $U_{* i}^{(0)}\left(=\left(T_{i}^{(0)} / e B_{0}\right) \partial \ln N^{(0)} / \partial r\right)$ is the ion poloidal diamagnetic drift velocity. Finally, Eq. (33) for circular cross section geometry can be written as

$$
\begin{aligned}
& {\left[4 U_{E}^{(0)}+\left(\frac{3}{2} \eta_{i}-1\right) U_{* i}^{(0)}+4\left(\frac{B_{\theta}}{B_{\phi}}\right) U_{\| i}^{(0)}\right] \frac{\partial n^{(1)}}{\partial \theta}} \\
& +5 \epsilon \eta_{i} U_{* i}^{(0)} \sin \theta \\
& \quad+\frac{3}{2}\left(U_{E}^{(0)}+\frac{B_{\theta}}{B_{\phi}} U_{\| i}^{(0)}\right)\left(\frac{m_{i} \nu_{c x}^{(0)} U_{\| i}^{(0)}}{T_{i}^{(0)}} \Delta_{1} q R\right) \cos \theta \\
& +\chi_{\| i}^{(0)}\left(\frac{m_{i} \nu_{c x} \Delta_{1}}{T_{i}^{(0)}}\right)\left(\frac{r}{q R}\right) U_{\| i}^{(0)} \sin \theta \\
& =2 \chi_{\| i}^{(0)}\left(\frac{r}{q^{2} R^{2}}\right) \frac{\partial^{2} n^{(1)}}{\partial \theta^{2}}+\frac{3}{2} r \Delta_{1} \nu_{c x}^{(0)} \cos \theta .
\end{aligned}
$$

We have replaced $\chi_{\| i}=3.9 T_{i}^{(0)} / m_{i} \nu_{i}^{(0)}, \quad \partial b^{(1)} / \partial \theta=\epsilon \sin \theta$ and $g(\theta)=\Delta_{1} \cos \theta$. Writing the solution of Eq. (37) in form of

$$
\begin{aligned}
& \frac{\partial\left(m_{i} N_{i}^{(0)} U_{\phi i}^{(0)}\right)}{\partial t}+m_{i} N_{i}^{(0)} \nu_{c x} U_{\phi i}^{(0)}+\frac{\partial}{\partial r}\left(m_{i} N_{i}^{(0)}\left\langle\tilde{u}_{E r} \tilde{u}_{\phi i}\right\rangle\right) \\
& =\frac{m_{i} B_{\phi}^{2}}{e r} \frac{\partial}{\partial r}\left\{-2 \frac{P_{i}^{(0)}}{B_{0}^{3}} \frac{B_{\phi}}{B_{\theta}}\left[3 U_{E}^{(0)}+\left(\eta_{i}-1\right) U_{* i}^{(0)}+3 \frac{B_{\theta}}{B_{\phi}} U_{\| i}^{(0)}\right] \oint n^{(1)} \frac{\partial b^{(1)}}{\partial \theta} \frac{d \theta}{2 \pi}+3.2 \frac{\chi_{\| i}^{(0)}}{q R} \frac{P_{i}^{(0)}}{B_{0}^{3}}\right. \\
& \left.\quad \times \oint \frac{\partial n^{(1)}}{\partial \theta} \frac{\partial b^{(1)}}{\partial \theta} \frac{d \theta}{2 \pi}+\frac{12}{10} \frac{P_{i}^{(0)}}{B_{0}^{2}} \frac{\nu_{i}^{(0)}}{\Omega_{i}^{(0)}} \frac{r}{B_{\phi 0}} \frac{\partial U_{\| i}^{(0)}}{\partial r}\right\}+\frac{m_{i} B_{\phi}^{2}}{e r}\left(m_{i} \nu_{c x}^{(0)} \Delta_{1} q R\right) \frac{\partial}{\partial r}\left\{\left(\frac{U_{\| i}^{(0)}}{T_{i}^{(0)}} \frac{P_{i}^{(0)}}{B_{0}^{3}}\right)\right. \\
& \left.\quad \times\left[-\frac{B_{\phi}}{B_{\theta}}\left(U_{E}^{(0)}-U_{* i}^{(0)}+\frac{B_{\theta}}{B_{\phi}} U_{\| i}^{(0)}\right) \oint \cos \theta n^{(1)} \frac{d \theta}{2 \pi}+\frac{B_{\phi}}{B_{\theta}}\left(2 U_{E}^{(0)}+2\left(1+\eta_{i}\right) U_{* i}^{(0)}+2 \frac{B_{\theta}}{B_{\phi}} U_{\| i}^{(0)}\right) \oint \cos \theta b^{(1)} \frac{d \theta}{2 \pi}\right]\right\} \\
& \quad-\frac{m_{i} B_{\phi}^{2}}{e r} \Delta_{1} q R \frac{\partial}{\partial r}\left\{\left(\frac{P_{i}^{(0)}}{B_{0}^{3}}\right)\left[\frac{\nu_{c x}^{(0)}}{\Omega_{i}}\left(\frac{\partial \ln N^{(0)}}{\partial r}\right)\left(\frac{B_{\phi}}{B_{\theta}} U_{\| i}^{(0)}\right)\left(1+\frac{\partial \ln U_{\| i}^{(0)}}{\partial \ln N^{(0)}}\right)\right] \oint\left(n^{(1)}+4 b^{(1)}\right) \cos \theta \frac{d \theta}{2 \pi}\right\},
\end{aligned}
$$

where in the above equation the relation $\oint\left(\sin \theta \partial f^{(1)} / \partial \theta\right) d \theta=-\oint \cos \theta f^{(1)} d \theta$ has been used. We use the value of $n^{(1)}$ from Eq. (38) and $b^{(1)}=-\epsilon \cos \theta$ to evaluate the integrals in Eq. (41). The equation providing the radial profile of toroidal velocity is then 


$$
\begin{aligned}
m_{i} \frac{\partial}{\partial t} N_{i} U_{\phi i}+\nu_{c x} m_{i} N_{i} U_{\phi i}+\frac{\partial}{\partial r}\left[m_{i} N_{i}\left(\left\langle\tilde{u}_{E} \tilde{u}_{\phi i}\right\rangle+U_{r i} U_{\phi i}\right)\right] \\
=\frac{m_{i} B_{\phi}^{2}}{e r} \frac{\partial}{\partial r}\left[\frac{\alpha_{2} \epsilon}{2}\left(-2 \frac{P_{i}^{(0)}}{B_{0}^{3}} \frac{B_{\phi}}{B_{\theta}}\right)\left[3 U_{E}^{(0)}+\left(\eta_{i}-1\right) U_{* i}^{(0)}+3 \frac{B_{\theta}}{B_{\phi}} U_{\| i}^{(0)}\right]-\frac{\alpha_{1} \epsilon}{2}\left(3.2 \frac{\chi_{\| i}^{(0)}}{q R} \frac{P_{i}^{(0)}}{B_{0}^{3}}\right)+\frac{12}{10} \frac{P_{i}^{(0)}}{B_{0}^{2}} \frac{\nu_{i}^{(0)}}{\Omega_{i}^{(0)}} \frac{r}{B_{\phi 0}} \frac{\partial U_{\| i}^{(0)}}{\partial r}\right] \\
\quad-m_{i} \nu_{c x}^{(0)} \Delta_{1} q R \frac{m_{i} B_{\phi}^{2}}{e r} \frac{\partial}{\partial r}\left[( \frac { U _ { \| i } ^ { ( 0 ) } } { T _ { i } ^ { ( 0 ) } } \frac { P _ { i } ^ { ( 0 ) } } { B _ { 0 } ^ { 3 } } ) \left\{\frac{\alpha_{1}}{2} \frac{B_{\phi}}{B_{\theta}}\left(U_{E}^{(0)}-U_{* i}^{(0)}+\frac{B_{\theta}}{B_{\phi}} U_{\| i}^{(0)}\right)+\frac{\epsilon}{2} \frac{B_{\phi}}{B_{\theta}}\left(2 U_{E}^{(0)}+2\left(1+\eta_{i}\right) U_{* i}^{(0)}+2 \frac{B_{\theta}}{B_{\phi}} U_{\| i}^{(0)}\right)\right.\right. \\
\left.\left.\quad+\left(\frac{\alpha_{1}}{2}-\frac{4 \epsilon}{2}\right) \frac{B_{\phi}}{B_{\theta}} \frac{T_{i}^{(0)}}{e B_{0}} \frac{\partial \ln N^{(0)}}{\partial r}\left(1+\frac{\partial \ln U_{\| i}^{(0)}}{\partial \ln N^{(0)}}\right)\right\}\right],
\end{aligned}
$$

where $\eta_{i}=\partial_{r} \ln T_{i}^{(0)} / \partial_{r} \ln N^{(0)}$. Here the second term $\nu_{c x} U_{\phi, i}$, in the RHS of Eq. (42) represents the standard flow damping via charge exchange with cold recycling neutrals from the wall, third term corresponds to toroidal momentum transfer through background turbulence and neoclassical radial convection effects and the RHS of above equation results from the flux averaged toroidal component of $\boldsymbol{\nabla} \cdot \stackrel{\leftrightarrow}{\pi}$ including the effects of asymmetric neutral gas fueling. We further simplify Eq. (42) to large aspect ratio tokamak plasmas, i.e., $L_{\perp} \ll r \ll R$ with circular cross section and discuss the relevance of the theory to some experiments in next section.

\section{APPLICATIONS OF THE THEORY AND DISCUSSION}

Equation (42) for $U_{\phi i}^{(0)}$ can be simplified further for specific experiments, namely, COMPASS-D and TEXTOR edge plasma parameters.

(1) In COMPASS-D (Refs. 4 and 5) the edge plasma parameters are typically $T_{i}^{(0)}=50 \mathrm{eV}, N_{i}^{(0)}=2 \times 10^{19} \mathrm{~m}^{-3}$ and $B_{0}=1.2 \mathrm{~T}, \quad I_{p}=0.19 \mathrm{MA}, \quad R_{0}=0.56 \mathrm{~m}, \quad a=0.17 \mathrm{~m}$, $\epsilon=0.3, \kappa($ elongation $)=1.7, \delta$ (triangularity $)=0.4$. The ion thermal velocity is thus $c_{i} \sim 7 \times 10^{4} \mathrm{~m} / \mathrm{s}$, whereas $\Omega_{i} \sim 1.2 \times 10^{8} \mathrm{~s}^{-1}, \quad a_{i} \sim 5.833 \times 10^{-4} \mathrm{~m}, \quad \nu_{i}$ $=Z_{\text {eff }} N_{i}^{(0)}\left(\mathrm{m}^{-3}\right) 10^{-12} / 2^{0.5} A_{i}^{0.5} T_{i}^{1.5}(\mathrm{eV}) \sim 4 \times 10^{4} \mathrm{~s}^{-1}$ and $\hat{\nu}_{i}$ (collisionality parameter) $=q R \nu_{i} / c_{i} \approx 1$. Here we assume $Z_{\text {eff }}=1$ and $A_{i}=1$ (hydrogen plasma). The safety factor $q_{a}$ is calculated from a known formula ${ }^{23}$

$$
\begin{aligned}
& q_{a}=\frac{5}{2} \frac{a^{2} B_{0}}{R I_{\mathrm{MA}}} G, \\
& G=\left[1+\kappa^{2}\left(1+2 \delta^{2}-1.2 \delta^{3}\right)\right]\left(\frac{1.17-0.65 \epsilon}{\left(1 .-\epsilon^{2}\right)^{2}}\right)
\end{aligned}
$$

( $G$ is a geometrical factor), which yields $q_{a} \sim 4.5$.

(2) TEXTOR edge parameters in the Radiative Improved Confinement Mode (Ref. 24) are: $T_{i} \approx 50 \mathrm{eV}, N_{i}^{(0)} \approx 2$ $\times 10^{19} \mathrm{~m}^{-3}$, and $B_{0}=2.25 \mathrm{~T}, I_{p}=400 \mathrm{kA}, R_{0}=1.75 \mathrm{~m}$, $a=0.46 \mathrm{~m}, \epsilon=0.26, q_{a}=3.4$. Thus $c_{i} \sim 5 \times 10^{4} \mathrm{~m} / \mathrm{s}, \Omega_{i}$ $\sim 1.12 \times 10^{8} \mathrm{~s}^{-1}, \quad a_{i} \sim 4.464 \times 10^{-4} \mathrm{~m}, \quad \nu_{i} \sim 8 \times 10^{4} \mathrm{~s}^{-1}$ (we take $Z_{\text {eff }}=2$ and $A_{i}=2$ ), and $\hat{\nu}_{i} \geqslant 1$.

Neoclassical theory ${ }^{15}$ in the high collision (Pfirsch-
Schlüter) regime predicts the value of poloidal flow $U_{\theta i}$ $\approx-1.83\left(T_{i} / e B_{\phi}\right)\left(\partial \ln T_{i} / \partial r\right)=-1.83 \eta_{i} U_{* i}$ (a brief outline of the derivation of $U_{\theta i}$ is given in Appendix B). The ratio of $Q$ to $S$ is

$$
\frac{Q}{S} \sim 0.64\left(1+2 \eta_{i}\right) \frac{\rho_{i}}{L_{N}} \frac{q R}{r} \frac{\nu_{i} q R}{c_{i}},
$$

where $Q=-5\left(1+1.964 \eta_{i}\right) U_{* i} \quad$ and $\quad S=7.8\left(c_{i}^{2} / \nu_{i} q R\right)$ $\times(r / q R)$. Introducing the COMPASS-D and TEXTOR edge plasma parameters, we find $Q / S<1$. In the limits $Q / S<1$ and $\left(U_{\| i}^{(0)} U_{* i}^{(0)} / c_{i}^{2}\right)<(r / q R)$, and $\nu_{c x}^{(0)}$ is slowly varying function of $r$, Eq. (42) can be simplified into

$$
\begin{aligned}
m_{i} \frac{\partial}{\partial t} N_{i} U_{\phi i}+\nu_{c x} m_{i} N_{i} U_{\phi i}+\frac{\partial}{\partial r}\left[m_{i} N_{i}\left(\left\langle\tilde{u}_{E} \tilde{u}_{\phi i}\right\rangle+U_{r i} U_{\phi i}\right)\right] \\
=\frac{\partial}{\partial r}\left[\eta_{2 i}\left(1+1.67 q \Delta_{1} \frac{\nu_{c x}}{\nu_{i}} \frac{B_{\phi}}{B_{\theta}}\right) \frac{\partial U_{\| i}^{(0)}}{\partial r}\right. \\
+0.2 q^{2} \frac{T_{i}^{(0)}}{e B_{\theta}}\left(\frac{\partial \ln T_{i}^{(0)}}{\partial r}\right)^{2} \\
\left.\quad \times\left(1-\frac{5.1 \Delta_{1}}{q} \frac{\nu_{c x}}{\nu_{i}} \frac{B_{\theta}}{B_{\phi}}\left(\frac{a_{i} \partial \ln T_{i}^{(0)}}{\partial r}\right)^{-2}\right)\right],
\end{aligned}
$$

where $\eta_{1 i}=3 P_{i} \nu_{i} / 10 \Omega_{i}^{2}$ is classical perpendicular viscosity coefficients ${ }^{18}$ and the terms proportional to $\nu_{c x}^{2}$ are smaller in comparison with the terms proportional to $\nu_{c x}$, and have been neglected in the derivation of Eq. (45). Note that Eq. (45) is a partial differential equation for the angular momentum density, which is first order with respect to $\partial / \partial t$ and second order with respect to $\partial / \partial r$, similar to a diffusive equation.

The analytical solution of radial transport diffusive Eq. (45) is complicated and more involved. In order to estimate the magnitude of toroidal flow of ions we neglect the momentum flux driven by background turbulence, toroidal momentum transfer due to neoclassical radial convection effects and the standard charge exchange damping with neutrals due to recycling from wall. The stationary (i.e., $\partial / \partial t \rightarrow 0$ ) equation for $U_{\phi i}^{(0)}$ is then 


$$
\begin{aligned}
(1+ & \left.1.67 q \Delta_{1} \frac{\nu_{c x}}{\nu_{i}} \frac{B_{\phi}}{B_{\theta}}\right) \frac{\partial U_{\| i}^{(0)}}{\partial r} \\
= & -0.2 q^{2} \frac{T_{i}^{(0)}}{e B_{\theta}}\left(\frac{\partial \ln T_{i}^{(0)}}{\partial r}\right)^{2} \\
\times & {\left[1-\frac{5.1 \Delta_{1}}{q} \frac{\nu_{c x}}{\nu_{i}} \frac{B_{\theta}}{B_{\phi}}\left(\frac{a_{i} \partial \ln T_{i}^{(0)}}{\partial r}\right)^{-2}\right] . }
\end{aligned}
$$

We note that in the absence of a beam, externally drawn polarization current and poloidal asymmetric neutral source $\left(\Delta_{1}=0\right)$, we recover the results of Claassen et al. [see Eq. (1) of Ref. 15] for the toroidal flow:

$$
\begin{aligned}
\frac{\partial U_{\| i}^{(0)}}{\partial r} & =0.107 q^{2} \frac{\partial \ln T_{i}^{(0)}}{\partial r} \frac{B_{\phi}}{B_{\theta}} U_{\theta i} \\
& \approx-0.2 q^{2} \frac{T_{i}^{(0)}}{e B_{\theta}}\left(\frac{\partial \ln T_{i}^{(0)}}{\partial r}\right)^{2}
\end{aligned}
$$

if $Q / S<1$ and $U_{\theta i}=-1.83\left(T_{i}^{(0)} / e B_{\phi}\right)\left(\partial \ln T_{i}^{(0)} / \partial r\right)$.

From Eq. (46), we first estimate the toroidal flow rotation in the presence of asymmetric gas fueling, i.e., $\Delta_{1} \neq 0$.

We solve Eq. (46) using local analysis and assuming the temperature scale length and toroidal velocity shear scale length are of the same order, viz.,

$$
\frac{1}{U_{\phi, i}} \frac{\partial U_{\phi, i}}{\partial r} \approx \frac{1}{T_{i}} \frac{\partial T_{i}}{\partial r} \approx-\frac{1}{L_{T, U_{\phi}}} .
$$

Note that we may identify $U_{\| i} \sim U_{\phi, i}$ since $U_{\theta, i} B_{\theta}$ $\ll U_{\phi, i} B_{\phi}$ in tokamak.

Now, Eq. (46) can be simplified to

$$
\begin{aligned}
(1+ & \left.1.67 q \Delta_{1} \frac{\nu_{c x}}{\nu_{i}} \frac{B_{\phi}}{B_{\theta}}\right)\left(\frac{U_{\| i}^{(0)}}{c_{i}}\right) \\
& =-0.2 q^{2}\left[\frac{\left(a_{i}\right)_{p}}{L_{T}}\right]\left[1-\frac{5.1 \Delta_{1}}{q} \frac{\nu_{c x}}{\nu_{i}} \frac{B_{\phi}}{B_{\theta}} \frac{L_{T}^{2}}{\left(a_{i}\right)_{p}^{2}}\right],
\end{aligned}
$$

where $\left(a_{i}\right)_{p} \equiv\left(B_{\phi} / B_{\theta}\right) a_{i}$ is the ion Larmor radius at poloidal magnetic field.

In order to estimate the value of asymmetric parameter $\left(\Delta_{1}\right)$, we assume the wall is saturated with neutrals and in steady state the inward neutral flux originating from the SOL is balanced by an outward ion flux. Thus

$$
N_{n} U_{n} \times 2 \pi R \times 2 \pi r=\frac{\left\langle N_{i}\right\rangle}{\tau_{p}} \times 2 \pi R \times \pi r^{2} \equiv N^{\prime} .
$$

Here, $\tau_{p}$ is the particle confinement time, $\left\langle N_{i}\right\rangle$ is the line averaged plasma density, $N_{n}$ and $U_{n}$ are the density and flow velocity of neutrals emerging from the SOL, and $R(r)$ is the major (minor) radius of plasma. The right-hand side of Eq. (50) represents the number of particles per second $(N)$ coming out from last closed plasma surface. Assuming the particle confinement time $\tau_{p} \sim 30 \times 10^{-3} \mathrm{~s}$, we get $N^{\prime}=3.2$ $\times 10^{21}$ particles/s for COMPASS-D and $N^{\prime}=5$ $\times 10^{22}$ particles/s for TEXTOR tokamaks. In both experiments, the gas-fueling rate ranges from (0.1-1.0) $\times 10^{20}$ particles/s. Thus the asymmetric parameter $\Delta_{1}$ varies from $\sim(0.5-5) \times 10^{-3}$. Equation (50) yields the neutral density at the edge $\left(N_{n}\right)_{\text {TEXTOR }} \approx 6 \times 10^{16} \mathrm{~m}^{-3}$ and $\left(N_{n}\right)_{\text {COMPASS-D }} \approx 1.6 \times 10^{16} \mathrm{~m}^{-3}$ if $U_{n} \sim c_{i} / 2$. The other parameters, which are useful in evaluation of toroidal flow, may be taken from the experiment as (i) $\left(B_{\phi} / B_{\theta}\right) \sim 12$, $\nu_{c x} / \nu_{i} \sim 2.3 \times 10^{-2}$ for TEXTOR and $\left(B_{\phi} / B_{\theta}\right) \sim 5.4$, $\nu_{c x} / \nu_{i} \sim 10^{-2}$ for COMPASS-D. When there is no gas puffing (i.e., $\left.\Delta_{1}=0\right)$ and assuming $q^{2}\left(a_{i}\right)_{p} / L_{T} \sim 1$, the toroidal velocity inside the LCFS is found to be $U_{\phi, i} \sim 0.2 c_{i}$ $\sim 10 \mathrm{~km} / \mathrm{s}$. In both machines the typical value of parameter $q\left(\nu_{c x} / \nu_{i}\right)\left(B_{\phi} / B_{\theta}\right)$ is of order $\sim 1, q^{2}\left(a_{i}\right)_{p} / L_{T} \sim 1$, and $\Delta_{1}$ $\ll 1$. The toroidal velocity predicted by the approximate analytical model is

$$
U_{\phi, i} \approx 0.2 \times\left(1-5.1 q^{2} \Delta_{1}\right) c_{i}
$$

where $\Delta_{1}=-\Delta_{1}$ for inboard side puffing and $\Delta_{1}=\Delta_{1}$ for outboard gas puffing.

For an asymmetric parameter $\Delta_{1} \sim 2 \times\left(1 / 5 q^{2}\right)$, Eq. (51) predicts a significant enhanced toroidal flow velocity in the edge if the gas fueling is applied from mid-plane of inboard side of tokamak. From Eq. (51). The value of toroidal flow velocity is $30 \mathrm{~km} / \mathrm{s}$ and according to Eq. (2), the radial electric field and its shear are also modified. It is interesting to note that for a fixed gas fueling rate the asymmetric parameter $\Delta_{1}$ is small in a machine like TEXTOR and is large in COMPASS-D. Thus the role of asymmetric gas is effectively stronger in COMPASS-D in comparison to TEXTOR. We also note that the sign of toroidal velocity shear $\left(\partial U_{\phi i}^{(0)} / \partial r\right)$ changes, for $\Delta_{1} \nu_{c x}^{*}<1$ and $\left(5.1 \Delta_{1} \nu_{c x}^{*} a_{i p}^{2} / q^{2} L_{T i}^{2}\right)>1$ (where $\left.\nu_{c x}^{*}=q \nu_{c x} B_{\phi} / \nu_{i} B_{\theta}\right)$, if the gas puff is switched from outboard to inboard side.

In conclusion, the effects of poloidal variation of neutral atoms on generation of neoclassical toroidal flow velocity in high collisionality plasma have been studied. The poloidal variation of neutral atoms associated with ion momentum damping and ion energy loss due to charge-exchange processes is included. The role of ionization neutral is neglected presently. The detail calculations including asymmetric ionization source in continuity equation will be presented in forthcoming paper. Recently Simakov and Catto ${ }^{25}$ have studied the effects of poloidal variation of neutral density on neoclassical toroidal flow. They have included effects due to neutral viscosity and ionization physics and conclude that the effects due to ionization are relatively small. In this paper, a simple poloidal variation of neutral source of the form $N_{n}$ $=N_{0 n}\left(1+\Delta_{1} \cos \theta\right)$ is assumed. Thus an explicit $\theta^{*}$-dependence similar to Füllöp et al. ${ }^{22}$ does not show up in the final toroidal flow equation (45).

\section{ACKNOWLEDGMENT}

The stay of Raghvendra Singh in Jülich was financed in the framework of the bilateral agreement between the Republic of India and Federal Republic of Germany in the fields of scientific and Technology development (Project No. IND 01/ 012). 


\section{APPENDIX A: THE STRESS TENSORS $\left(\stackrel{\leftrightarrow}{\pi}_{i}\right)$}

In the limit $\nu_{i} / \Omega_{i} \ll 1$, the stress tensor $^{18}$ with Mikhailovskii and Tsypin ${ }^{19}$ correction can be split into three parts. The off-diagonal or parallel stress tensor is given as ${ }^{15}$

$$
\begin{aligned}
\stackrel{\pi}{\pi}_{0 i}= & -3 \eta_{0 i}\left(\hat{n} \hat{n}-\frac{\overleftrightarrow{I}}{3}\right)\left[\hat{n} \cdot \boldsymbol{\nabla} \mathbf{U}_{i} \cdot \hat{n}-\frac{\boldsymbol{\nabla} \cdot \mathbf{U}_{i}}{3}\right. \\
& +1.615 \frac{2}{5 P_{i}}\left(\hat{n} \cdot \boldsymbol{\nabla} \mathbf{q}_{i} \cdot \hat{n}-\frac{\boldsymbol{\nabla} \cdot \mathbf{q}_{i}}{3}\right)+0.615 \times \frac{2}{5 P_{i}} \\
& \times\left\{-\left(\hat{n} \cdot \boldsymbol{\nabla} \mathbf{q}_{i}^{*} \cdot \hat{n}-\frac{\boldsymbol{\nabla} \cdot \mathbf{q}_{i}^{*}}{3}\right)+\frac{\mathbf{q}_{i} \cdot \boldsymbol{\nabla} \ln P_{i}}{3}\right. \\
& \left.\left.-\frac{\left(2 \mathbf{q}_{i}-\mathbf{q}_{i}^{*}\right) \cdot \boldsymbol{\nabla} \ln T_{i}}{3}\right\}\right]
\end{aligned}
$$

where $\eta_{0, i}=0.96 P_{i} \nu_{i}^{-1}$ and the heat fluxes are given by

$$
\begin{aligned}
\mathbf{q}_{i}= & -\frac{P_{i}}{m_{i}}\left[\frac{3.9}{\nu_{i}^{-1}} \hat{n} \hat{n} \cdot \nabla T_{i}-\frac{5}{2 \Omega_{i}} \hat{n} \times \nabla T_{i}\right. \\
& \left.-\frac{2 \nu_{i}}{\Omega_{i}^{2}} \hat{n}\left(\hat{n} \times \nabla T_{i}\right)\right],
\end{aligned}
$$

and

$$
\mathbf{q}_{i}^{*}=1.04 \frac{P_{i}}{m_{i} \nu_{i}} \hat{n} \hat{n} \cdot \nabla T_{i}
$$

The gyrostress tensor is given as

$$
\begin{aligned}
\overleftrightarrow{\pi}_{3-4, i}= & -\eta_{3, i}\left\{(\hat{p} \hat{p}-\hat{b} \hat{b})\left[\hat{b} \cdot \boldsymbol{\nabla} \mathbf{U}_{i} \cdot \hat{p}+\hat{p} \cdot \boldsymbol{\nabla} \mathbf{U}_{i} \cdot \hat{b}+\frac{2}{5 P_{i}}\left(\hat{b} \cdot \boldsymbol{\nabla} \mathbf{q}_{i} \cdot \hat{p}+\hat{p} \cdot \boldsymbol{\nabla} \mathbf{q}_{i} \cdot \hat{b}\right)\right]-(\hat{p} \hat{b}+\hat{b} \hat{p})\left[\hat{p} \cdot \boldsymbol{\nabla} \mathbf{U}_{i} \cdot \hat{p}\right.\right. \\
& \left.-\hat{b} \cdot \boldsymbol{\nabla} \mathbf{U}_{i} \cdot \hat{b}+\frac{2}{5 P_{i}}\left(\hat{p} \cdot \boldsymbol{\nabla} \mathbf{q}_{i} \cdot \hat{p}-\hat{b} \cdot \boldsymbol{\nabla} \mathbf{q}_{i} \cdot \hat{b}\right)\right]+2\left(\hat{p} \hat{n}+\hat{n} \hat{p}\left[\hat{b} \cdot \boldsymbol{\nabla} \mathbf{U}_{i} \cdot \hat{n}+\hat{n} \cdot \boldsymbol{\nabla} \mathbf{U}_{i} \cdot \hat{b}+\frac{2}{5 P_{i}}\left(\hat{b} \cdot \boldsymbol{\nabla} \mathbf{q}_{i} \cdot \hat{n}+\hat{n} \cdot \boldsymbol{\nabla} \mathbf{q}_{i} \cdot \hat{b}\right)\right]\right. \\
& -2(\hat{b} \hat{n}+\hat{n} \hat{b})\left[\hat{p} \cdot \boldsymbol{\nabla} \mathbf{U}_{i} \cdot \hat{n}+\hat{n} \cdot \boldsymbol{\nabla} \mathbf{U}_{i} \cdot \hat{p}+\frac{2}{5 P_{i}}\left(\hat{p} \cdot \boldsymbol{\nabla} \mathbf{q}_{i} \cdot \hat{n}+\hat{n} \cdot \boldsymbol{\nabla} \mathbf{q}_{i} \cdot \hat{p}\right)\right],
\end{aligned}
$$

where $\eta_{3, i}=P_{i} / \Omega_{i}$.

The perpendicular stress tensor is

$$
\begin{aligned}
\pi_{1-2, i}= & -\eta_{1, i}\left\{(\hat{p} \hat{p}-\hat{b} \hat{b})\left[\hat{p} \cdot \boldsymbol{\nabla} \mathbf{U}_{i} \cdot \hat{p}-\hat{b} \cdot \boldsymbol{\nabla} \mathbf{U}_{i} \cdot \hat{b}+\frac{2}{5 P_{i}}\left(\hat{p} \cdot \boldsymbol{\nabla} \mathbf{q}_{i} \cdot \hat{p}-\hat{b} \cdot \boldsymbol{\nabla} \mathbf{q}_{i} \cdot \hat{b}\right)\right]+(\hat{p} \hat{b}+\hat{b} \hat{p})\left[\hat{p} \cdot \boldsymbol{\nabla} \mathbf{U}_{i} \cdot \hat{b}\right.\right. \\
& \left.+\hat{b} \cdot \boldsymbol{\nabla} \mathbf{U}_{i} \cdot \hat{p}+\frac{2}{5 P_{i}}\left(\hat{p} \cdot \boldsymbol{\nabla} \mathbf{q}_{i} \cdot \hat{b}+\hat{b} \cdot \boldsymbol{\nabla} \mathbf{q}_{i} \cdot \hat{p}\right)\right]+4(\hat{p} \hat{n}+\hat{n} \hat{p})\left[\hat{p} \cdot \boldsymbol{\nabla} \mathbf{U}_{i} \cdot \hat{n}+\hat{n} \cdot \boldsymbol{\nabla} \mathbf{U}_{i} \cdot \hat{p}+\frac{2}{5 P_{i}}\left(\hat{p} \cdot \boldsymbol{\nabla} \mathbf{q}_{i} \cdot \hat{n}+\hat{n} \cdot \boldsymbol{\nabla} \mathbf{q}_{i} \cdot \hat{p}\right)\right] \\
& \left.+4(\hat{b} \hat{n}+\hat{n} \hat{b})\left[\hat{b} \cdot \boldsymbol{\nabla} \mathbf{U}_{i} \cdot \hat{n}+\hat{n} \cdot \boldsymbol{\nabla} \mathbf{U}_{i} \cdot \hat{b}+\frac{2}{5 P_{i}}\left(\hat{b} \cdot \boldsymbol{\nabla} \mathbf{q}_{i} \cdot \hat{n}+\hat{n} \cdot \boldsymbol{\nabla} \mathbf{q}_{i} \cdot \hat{b}\right)\right]\right\},
\end{aligned}
$$

where $\eta_{1 i}=3 P_{i} \nu_{i} / 10 \Omega_{i}^{2}$, the index $1-2$ refers to Braginskii's coefficients ${ }^{18}$ and $\eta_{2 i}=4 \eta_{1 i}$.

\section{APPENDIX B: OUT LINE OF THE DERIVATION OF POLOIDAL FLOW $\left(U_{\theta i}\right)$}

In this appendix, we derive the expression for poloidal flow $\left(U_{\theta i}\right)$ without an asymmetric neutral source. We use two-fluid equations including the Mikhailovskii-Tsypin ${ }^{19}$ corrections to the Braginskii's ${ }^{18}$ stress tensors. We show the importance of these corrections to the Braginskii's ${ }^{18}$ stress tensors for reproducing the expression of neoclassical poloidal flow $\left(U_{\theta i}\right)$ which is close to the expression derived by Hazeltine $^{12}$ by using a kinetic analysis for high collisionality regime. We also show that the poloidal flow $U_{\theta i}=0$, if the Mikhailovskii-Tsypin corrections to the Braginskii's stress tensors are absent.

Integrating the sum of the parallel ion and electron mo- mentum equations over poloidal angle after multiplying by $J B$, we get

$$
\oint J B \frac{d \chi}{2 \pi}\left[\boldsymbol{\nabla} \cdot \stackrel{\leftrightarrow}{\pi}+m_{i} N_{i}\left(\frac{\partial}{\partial t}+\mathbf{U}_{i} \cdot \boldsymbol{\nabla}\right) \mathbf{U}_{i}\right] \cdot \hat{n}=0
$$

In the limit $\left(\nu_{i} / \Omega_{i}\right)\left(q^{2} R^{2} / r L_{\psi}\right) \ll 1$, the contribution from the gyrostress tensor (i.e., $\overleftrightarrow{\pi}_{-, i}$, and $\overleftrightarrow{\pi}_{1-2, i}$ ) to Eq. (B1) can be neglected. For simplicity neglecting the inertia term, Eq. (B1) can be written as

$$
\begin{aligned}
\oint J B \frac{d \chi}{2 \pi}\left(\boldsymbol{\nabla} \cdot \stackrel{\pi}{\pi, i}_{0}\right) \cdot \hat{n}= & -\oint J B \frac{d \chi}{2 \pi}\left[\hat{p} \cdot \nabla \hat{n} \cdot \hat{p} \pi_{0 \psi, \psi}\right. \\
& +\hat{b} \cdot \boldsymbol{\nabla} \hat{n} \cdot \hat{b} \pi_{0 \beta, \beta} \\
& -\oint \frac{d \chi}{2 \pi} \frac{\partial B}{\partial \chi} h_{\phi} h_{\psi} \frac{B}{B} \pi_{0\|,\|}=0
\end{aligned}
$$


where the stress tensor $\overleftrightarrow{\pi}_{0, i}$ including the MikhailovskiiTsypin corrections is given as

$$
\begin{aligned}
\overleftrightarrow{\pi}_{0 i}= & -3 \eta_{0 i}\left(\hat{n} \hat{n}-\frac{\overleftrightarrow{I}}{3}\right)\left[\hat{n} \cdot \boldsymbol{\nabla} \mathbf{U}_{i} \cdot \hat{n}-\frac{\boldsymbol{\nabla} \cdot \mathbf{U}_{i}}{3}+1.615\right. \\
& \times \frac{2}{5 P_{i}}\left(\hat{n} \cdot \boldsymbol{\nabla} \mathbf{q}_{i} \cdot \hat{n}-\frac{\boldsymbol{\nabla} \cdot \mathbf{q}_{i}}{3}\right)+0.615 \times \frac{2}{5 P_{i}} \\
& \times\left\{-\left(\hat{n} \cdot \boldsymbol{\nabla} \mathbf{q}_{i}^{*} \cdot \hat{n}-\frac{\boldsymbol{\nabla} \cdot \mathbf{q}_{i}^{*}}{3}\right)+\frac{\mathbf{q}_{i} \cdot \boldsymbol{\nabla} \ln P_{i}}{3}\right. \\
& \left.\left.-\frac{\left(2 \mathbf{q}_{i}-\mathbf{q}_{i}^{*}\right) \cdot \boldsymbol{\nabla} \ln T_{i}}{3}\right\}\right],
\end{aligned}
$$

where $\eta_{0 i}=0.96 P_{i} \nu_{i}^{-1}$ is the parallel ion viscous coefficient, $\mathbf{q}_{i}$ is the standard ion heat flux as given in Braginskii's energy equation [ ], $\mathbf{q}_{i}^{*}=1.04\left(P_{i} / m_{i} \nu_{i}\right) \hat{n} \hat{n} \cdot \boldsymbol{\nabla} T_{i}$ and it is easily seen from Eq. (B3) that $\pi_{\psi, \psi}=\pi_{\beta, \beta}=-(1 / 2) \pi_{\|,\|}$. The expressions in Eq. (B2) with Christoffel symbols $(\hat{p}, \hat{b}, \hat{n})$ are related to flux coordinates $(\psi, \chi, \phi)$ system through the following relations:

$$
\begin{aligned}
& J B(\hat{p} \cdot \nabla \hat{n} \cdot \hat{p}+\hat{b} \cdot \nabla \hat{n} \cdot \hat{b})=-\frac{1}{B} \frac{\partial B}{\partial \chi}, \\
& h_{\psi}=1 / h_{\phi} B_{\chi} .
\end{aligned}
$$

Combining Eqs. (B2) and (B4), we get

$$
\oint J B \frac{d \chi}{2 \pi}\left(\nabla \cdot \stackrel{\pi}{0, i}_{i}\right) \cdot \hat{n}=-\frac{3}{2} \oint \frac{d \chi}{2 \pi} \frac{\partial \ln B}{\partial \chi} \pi_{0\|,\|}=0 .
$$

Employing the expression of $\pi_{0\|,\|}$ from Eq. (B3) into Eq. (B5), we get

$$
\begin{gathered}
\eta_{0 i} h_{\chi}^{-1}\left\{3 U_{\chi i}^{(0)} \oint\left(\frac{\partial b^{(1)}}{\partial \chi}\right)^{2}-\left[2 U_{E}^{(0)}-\left(4+\eta_{i}\right) U_{* i}^{(0)}\right.\right. \\
\left.+2 \frac{B_{\chi}^{(0)}}{B_{0}} U_{\| i}^{(0)} \oint \frac{\partial n^{(1)}}{\partial \chi} \frac{\partial b^{(1)}}{\partial \chi}\right] \\
-1.615 \eta_{i} U_{* i}^{(0)} \oint\left(\frac{\partial b^{(1)}}{\partial \chi}\right)^{2} \\
-5.549 h_{\chi}^{-1} \frac{T_{i}^{(0)}}{m_{i} \nu_{i}^{(0)}} \frac{B_{\chi}^{2}}{B_{\phi}^{2}} h_{\chi}^{-1} \oint \frac{\partial t_{i}^{(1)}}{\partial \chi} \frac{\partial b^{(1)}}{\partial \chi} \\
\left.-\oint \frac{\partial b^{(1)}}{\partial \chi}\left(-U_{* i}^{(0)} \frac{\partial t_{i}^{(1)}}{\partial \chi}+\eta_{i} U_{* i}^{(0)} \frac{\partial n^{(1)}}{\partial \chi}\right)\right\} \frac{d \chi}{2 \pi}=0 .
\end{gathered}
$$

In the limits $Q / S \approx\left(\nu_{i} / \Omega_{i}\right)\left(q^{2} R^{2} / r L_{\psi}\right) \ll 1$, Eq. (B4) can be written in leading order as

$$
\begin{aligned}
\left\{3 U_{\chi i}^{(0)} \oint\left(\frac{\partial b^{(1)}}{\partial \chi}\right)^{2}=\right. & 1.615 \eta_{i} U_{* i}^{(0)} \oint\left(\frac{\partial b^{(1)}}{\partial \chi}\right)^{2} \\
& +5.549 h_{\chi}^{-1} \frac{T_{i}^{(0)}}{m_{i} \nu_{i}^{(0)}} \frac{B_{\chi}^{2}}{B_{\phi}^{2}} h_{\chi}^{-1} \\
& \left.\times \oint \frac{\partial^{2} t_{i}^{(1)}}{\partial \chi^{2}} \frac{\partial b^{(1)}}{\partial \chi}\right\} \frac{d \chi}{2 \pi} .
\end{aligned}
$$

It is interesting to note here that right-hand side of Eq. (B7) is only due to the Mikhailoskii and Tsypin correction to the Braginskii stress tensor. If this correction is absent in the Braginskii stress tensor, then one will not be able to recover the expression for the poloidal flow $\left(U_{\theta i} \approx-1.83 \eta_{i} U_{* i}\right)$.

Using Eqs. (34) and (39), the above integrals can be written as

$$
\begin{aligned}
& \oint\left(\frac{\partial b^{(1)}}{\partial \chi}\right)^{2} \frac{d \chi}{2 \pi}=\frac{\epsilon}{2}, \\
& \oint \frac{\partial^{2} t_{i}^{(1)}}{\partial \chi^{2}} \frac{\partial b^{(1)}}{\partial \chi} \frac{d \chi}{2 \pi}=\epsilon \alpha_{2},
\end{aligned}
$$

where the relation $t_{i}^{(1)} \sim-2 n^{(1)}$ from Eq. (36) is used. In the limit $Q / S \ll 1$, the expression for $\alpha_{2}$ is given as

$$
\alpha_{2} \approx-\frac{Z}{S}=-\frac{5 \epsilon \eta_{i} U_{* i}}{2 \chi_{\| i}^{(0)}}\left(\frac{q^{2} R^{2}}{r}\right) ; \quad \chi_{\| i}^{(0)}=3.9 \frac{T_{i}^{(0)}}{m_{i} \nu_{i}} .
$$

A final expression for the poloidal flow $\left(U_{\theta i}\right)$ can be obtained by combining Eqs. (B7)-(B9), and it would follow that

$$
U_{\theta i}=-1.84 \frac{T_{i}^{(0)}}{e B_{0}} \frac{\partial \ln T_{i}^{(0)}}{\partial r} .
$$

${ }^{1}$ B. A. Carreras, L. W. Owen, R. Maingi, P. K. Mioduszewski, T. N. Carlstrom, and R. J. Groebner, Phys. Plasmas 5, 2623 (1998).

${ }^{2}$ R. L. Boivin, J. A. Goetz, A. E. Hubbard et al., Phys. Plasmas 7, 1919 (2000).

${ }^{3}$ P. Gohil, L. R. Bayrol, T. C. Jernigan, K. H. Burrell, and T. N. Carlstrom, Phys. Rev. Lett. 86, 644 (2001).

${ }^{4}$ M. Valoviè, S. J. Fielding, P. G. Carolan, B. Lloyd, M. Price, V. Shevchenko, S. You, and M. J. Walsh, Contributed Papers, 28th EPS Conference on Controlled Fusion and Plasma Physics, Maderia Tecnopolo, 2001, Vol. 25A, 1829.

${ }^{5}$ M. Valoviè, P. G. Carlon, A. R. Frield, S. J. Fielding et al., Plasma Phys. Controlled Fusion 44, A175 (2002).

${ }^{6}$ A. R. Field, P. G. Carolan, R. J. Arends et al., Plasma Phys. Controlled Fusion 44, A113 (2002).

${ }^{7}$ B. Coppi, M. N. Rosenbluth, and R. Z. Sagdeev, Phys. Fluids 10, 582 (1967).

${ }^{8}$ B. N. Rogers, J. F. Drake, and A. Zeiler, Phys. Rev. Lett. 81, 4396 (1998). ${ }^{9}$ H. Biglari, P. H. Diamond, and P. W. Terry, Phys. Fluids B 2, 1 (1990).

${ }^{10}$ P. W. Terry, Rev. Mod. Phys. 72, 109 (2000).

${ }^{11}$ A. Rogister, Phys. Plasmas 1, 619 (1994).

${ }^{12}$ R. D. Hazeltine, Phys. Fluids 17, 961 (1974).

${ }^{13}$ F. L. Hinton and R. D. Hazeltine, Rev. Mod. Phys. 48, 239 (1976).

${ }^{14}$ V. Rozhansky and M. Tendler, Phys. Fluids B 4, 1877 (1992).

${ }^{15}$ H. A. Claassen, H. Gerhauser, A. Rogister, and C. Yarim, Phys. Plasmas 7, 3699 (2000).

${ }^{16}$ A. L. Rogister, J. E. Rice, A. Nicolai, A. Ince-Cushman, and S. Gangadhara, Nucl. Fusion 42, 1144 (2002). 
${ }^{17}$ J. E. Rice, J. A. Goetz, R. S. Granetz et al., Phys. Plasmas 7, 1825 (2000).

${ }^{18}$ S. I. Braginskii, in Reviews of Plasma Physics, edited by M. A. Leontovich (Consultants Bureau, New York, 1965), Vol. 1, p. 214.

${ }^{19}$ A. B. Mikhailovskii and V. S. Tsypin, Beitr. Plasmaphys. 24, 335 (1984).

${ }^{20}$ P. Helander, S. I. Krasheninnikov, and P. J. Catto, Phys. Plasmas 1, 3174 (1994).
${ }^{21}$ P. J. Catto, P. Helender, J. W. Connor, and R. D. Hazeltine, Phys. Plasmas 5, 3961 (1998).

${ }^{22}$ T. Fülöp, P. Helander, and P. J. Catto, Phys. Rev. Lett. 89, 225003 (2002).

${ }^{23}$ W. M. Sracey, Contrib. Plasma Phys. 42, 283 (2002).

${ }^{24}$ G. Mank, A. M. Messiaen, J. Ongena et al., Phys. Rev. Lett. 85, 2312 (2000).

${ }^{25}$ A. N. Simakov and P. J. Catto, Phys. Plasmas 10, 398 (2003). 